\title{
Osteosíntesis en fracturas oblicuas o espiroideas largas de metacarpianos: comparación entre tratamiento con tornillos interfragmentarios solos y placa con tornillos
}

\author{
Ángel Ferrando, Guillermo F. Belluschi, Roberto Andreozzi, Juan Martín Perrone, Hugo Sarmiento, Eliana Petrucelli \\ Equipo de Cirugía de Mano y Miembro Superior, Servicio de Ortopedia y Traumatología, Complejo Médico Churruca-Visca, \\ Ciudad Autónoma de Buenos Aires, Argentina
}

\section{RESUMEN}

Objetivos: Comparar el tratamiento de pacientes con fracturas oblicuas o espiroideas largas de metacarpianos, mediante reducción abierta y fijación interna con tornillos interfragmentarios solos o placas y tornillos. Materiales y Métodos: Se realizó un estudio retrospectivo comparativo entre 2 grupos de pacientes: uno con 24 pacientes tratados con tornillos interfragmentarios solos y otro con 17 pacientes tratados mediante osteosíntesis con placas y tornillos. En ambos, se utilizó un abordaje longitudinal dorsal, y se les indicó inmovilización posoperatoria con valva de yeso y rehabilitación con el mismo equipo de terapistas ocupacionales. Tras un seguimiento mínimo de 12 meses, se evaluaron los resultados con el puntaje DASH, la movilidad activa total, la distancia pulpejo-palma y la fuerza con dinamometría comparativa. Se consideró significativo un valor $p<0,05$. Resultados: El tiempo promedio de seguimiento fue de 24.5 meses (rango 12-43). No se hallaron diferencias estadísticamente significativas en el puntaje DASH, la movilidad activa total, la distancia pulpejo-palma y la fuerza. El tiempo hasta el reingreso laboral fue inferior en el grupo tratado con placas y tornillos, aunque se registraron dos casos de retiro de material, sumado, en uno de ellos, a adherencia tendinosa (tenólisis). Conclusiones: El tratamiento de pacientes con fracturas oblicuas o espiroideas largas de metacarpianos, mediante tornillos interfragmentarios solos o placas y tornillos logró resultados similares, se destaca el menor tiempo hasta la reincorporación laboral y la mayor cantidad de complicaciones con placas y tornillos.

Palabras clave: Fractura; metacarpiano; osteosíntesis; placa; tornillos solos.

Nivel de Evidencia: III

Osteosynthesis in Long Oblique and Spiral Metacarpal Fractures: Comparison Between Interfragmentary Screws and Plating

\section{ABSTRACT}

Objective: To compare open reduction and internal fixation with interfragmentary screws and with plates and screws for the treatment of long oblique or spiral metacarpal fractures. Materials and Methods: A comparative retrospective study was carried out between 2 groups of patients treated surgically. In the first group, 24 patients were treated with interfragmentary screws and in the second group, 17 patients were treated with plate and screw osteosynthesis. A dorsal longitudinal approach was used. Postoperatively, immobilization was performed using a plaster splint; both groups followed rehabilitation with same occupational therapist team. After a minimum follow-up of 12 months, they were evaluated with the DASH score, total active motion (TAM), pulp-to-palm distance, and comparative contralateral dynamometry. A p value $<0.05$ was considered significant. Results: The average followup was 24.5 months (range 12-43 months). There were no statistically significant differences in DASH, TAM, pulp-to-palm distance, and strength. The group treated with plate and screw fixation returned to work earlier, although there were two cases of implant removal, in addition to tendon adherence (tenolysis) in one of them. Conclusions: The treatment of patients with long oblique or spiral metacarpal fractures with interfragmentary screws or plates and screws showed similar outcomes; the treatment with plate and screws allowed an earlier return to work but had a greater number of complications.

Keywords: Fracture; metacarpal; osteosynthesis; plate; screws alone.

Level of Evidence: III

Recibido el 16-4-2021. Aceptado luego de la evaluación el 8-5-2021 • Dr. ÁNGEL A. FERRANDO • aferrando@fibertel.com.ar

https://orcid.org/0000-0002-5182-3977

Cómo citar este artículo: Ferrando Á, Belluschi GF, Andreozzi R, Perrone JM, Sarmiento H, Petrucelli E. Osteosíntesis en fracturas oblicuas o espiroideas largas de metacarpianos: comparación entre tratamiento con tornillos interfragmentarios solos y placa con tornillos. Rev Asoc Argent Ortop Traumatol 2021;86(5):621-628. https://doi.org/10.15417/issn. 1852-7434.2021.86.5.1345 


\section{INTRODUCCIÓN}

Las fracturas de los huesos metacarpianos representan unas de las lesiones más frecuentes de la mano y provocan una limitación funcional transitoria o definitiva. ${ }^{1,2}$ La mayoría de estas fracturas se pueden tratar satisfactoriamente en forma incruenta, pero si hay un desplazamiento inaceptable, o son irreductibles o inestables, debe optarse por la cirugía. ${ }^{3-5}$ Existen múltiples sistemas de fijación ideados para tal fin: fijadores externos, clavijas percutáneas colocadas con distintos montajes, tornillos endomedulares, cerclaje de alambre, tornillos solos, placas y tornillos, entre otros. ${ }^{6-8,12}$

Las fracturas diafisarias de los metacarpianos se clasifican clásicamente en tres grupos: transversales, oblicuas (o espiroideas) y conminutas. ${ }^{12}$ La disposición del trazo, sumada a otras características de la lesión, como las lesiones de partes blandas asociadas, la presencia de fracturas múltiples, la exposición ósea, la edad del paciente, el compromiso de la mano dominante, los requerimientos funcionales y, en gran medida, las preferencias y la experiencia del cirujano, permitirán identificar el mejor método de tratamiento para cada lesión. ${ }^{4,7}$

Las fracturas oblicuas o espiroideas largas (de trazo simple, cuya longitud alcanza o supera el doble del diámetro de la diáfisis del metacarpiano afectado $)^{4}$ suelen requerir tratamiento quirúrgico, debido al cabalgamiento o la rotación que habitualmente presentan. $5,6,8,11,12$

El objetivo de este estudio fue comparar el tratamiento de pacientes con fracturas oblicuas o espiroideas largas de metacarpianos, mediante reducción abierta y fijación interna con tornillos interfragmentarios solos o con placas y tornillos.

\section{MATERIALES Y MÉTODOS}

Se realizó un estudio retrospectivo que evaluó a pacientes entre agosto de 2009 y diciembre de 2015. En nuestro Servicio, se trataron con cirugía 288 fracturas de metacarpianos en 255 pacientes. Los criterios de inclusión fueron: pacientes $>18$ años, con fracturas cerradas simples oblicuas o espiroideas largas de un único metacarpiano, con acortamiento $>3 \mathrm{~mm}$, vicio rotatorio con solapamiento digital distal o ángulo $>20^{\circ}$, sin antecedentes quirúrgicos y un seguimiento mínimo de 12 meses. Se excluyó a pacientes con fracturas asociadas de más de un metacarpiano u otro hueso de la mano, fractura expuesta, lesión asociada de partes blandas o un seguimiento inadecuado.

Se obtuvo una muestra final de 41 pacientes, divididos en dos grupos, según si habían sido tratados con tornillos interfragmentarios solos (grupo 1) u osteosíntesis con placas y tornillos (grupo 2), respectivamente. La elección de la técnica y del implante estuvo sujeta a la decisión del equipo tratante.

El grupo 1 estaba integrado por 24 pacientes (5 mujeres y 19 hombres), con una edad promedio de 41 años (rango 17-58) y compromiso de la mano derecha (15 casos) e izquierda (9 casos). La mano hábil estaba afectada en 17 casos y la no hábil, en siete. La cirugía se llevó a cabo, en promedio, a los ocho días de la fractura (rango 3-19). En estos pacientes, se utilizaron dos o tres tornillos de 1,5; 1,7; 2,0 o 2,3 mm de diámetro, con una técnica de compresión interfragmentaria (Tabla 1, Figura 1).

El grupo 2 estaba formado por 17 pacientes ( 3 mujeres y 14 hombres), con una edad promedio de 41.8 años (rango 17-71) y compromiso de la mano derecha (12 casos) e izquierda (5 casos). Trece pacientes tenían compromiso de la mano hábil y cuatro, de la no hábil. La cirugía se realizó, en promedio, a los siete días de la fractura (rango 4-14). Se utilizaron placas no bloqueadas y tornillos de 2,0 y 2,3 mm (Tabla 2, Figura 2).

\section{Técnica quirúrgica}

Bajo bloqueo del plexo braquial guiado por ecografía y manguito hemostático, en todos los casos, se realizó un abordaje longitudinal dorsal en el espacio intermetacarpiano adyacente al metacarpiano afectado. Se efectuó una apertura longitudinal de los músculos intrínsecos y el periostio, elevándolos y separándolos del hueso en un único plano. A continuación, se procedió a la reducción y la osteosíntesis del metacarpiano con tornillos interfragmentarios (para instaurarlos debe cumplirse la premisa de que la longitud del trazo fracturario sea, al menos, dos veces el diámetro del metacarpiano y que el emplazamiento del orificio del tornillo sea perpendicular al trazo) o placa y tornillos, según la técnica AO. Se realizó el cierre de partes blandas con cobertura del implante, creando un plano entre este y los tendones extensores, y se colocó un vendaje estéril. 
Tabla 1. Serie de pacientes tratados con tornillos interfragmentarios solos

\begin{tabular}{|c|c|c|c|c|c|c|c|c|c|c|c|c|c|}
\hline $\mathbf{N}^{\circ}$ & Edad & Sexo & $\begin{array}{c}\text { Mano } \\
\text { lesionada }\end{array}$ & $\begin{array}{l}\text { Mano } \\
\text { hábil }\end{array}$ & $\begin{array}{l}\text { Meta- } \\
\text { carpiano } \\
\text { afectado }\end{array}$ & $\begin{array}{l}\text { Días } \\
\text { preq }\end{array}$ & $\begin{array}{c}\text { Dias de } \\
\text { inmovili- } \\
\text { zación }\end{array}$ & $\begin{array}{c}\% \\
\text { de } \\
\text { fuerza }\end{array}$ & $\begin{array}{c}\text { Distancia } \\
\text { pulpejo- } \\
\text { palma }\end{array}$ & $\begin{array}{c}\text { Puntaje } \\
\text { DASH }\end{array}$ & $\begin{array}{c}\text { Días hasta } \\
\text { el alta } \\
\text { laboral }\end{array}$ & $\begin{array}{c}\text { Movilidad } \\
\text { activa } \\
\text { total }\end{array}$ & Tornillos \\
\hline 1 & 31 & M & I & No & 3 & 6 & 12 & 100 & 0 & 1,2 & 68 & 260 & 2 \\
\hline 2 & 22 & M & D & Sí & 3 & 3 & 10 & 120,32 & 0 & 0 & 76 & 272 & 2 \\
\hline 3 & 29 & M & D & Sí & 4 & 9 & 14 & 97,14 & 0,5 & 2,16 & 52 & 265 & 2 \\
\hline 4 & 37 & M & I & No & 5 & 10 & 15 & 94,87 & 0 & 0 & 75 & 258 & 2 \\
\hline 5 & 58 & M & D & Sí & 2 & 12 & 17 & 78,5 & 1 & 4,51 & 83 & 240 & 2 \\
\hline 6 & 48 & M & I & No & 4 & 11 & 14 & 97,5 & 0 & 1,2 & 105 & 267 & 2 \\
\hline 7 & 32 & $\mathrm{~F}$ & D & Sí & 4 & 8 & 16 & 90 & 0 & 0 & 72 & 247 & 2 \\
\hline 8 & 39 & M & D & Sí & 3 & 5 & 15 & 94,74 & 0 & 0 & 77 & 263 & 2 \\
\hline 9 & 43 & M & I & Sí & 4 & 6 & 18 & 92,96 & 0 & 0,83 & 70 & 260 & 3 \\
\hline 10 & 32 & M & D & Sí & 4 & 7 & 15 & 97,3 & 0,8 & 1,76 & 84 & 230 & 3 \\
\hline 11 & 27 & M & D & Sí & 2 & 4 & 17 & 107,05 & 0 & 1,75 & 69 & 270 & 2 \\
\hline 12 & 29 & $\mathrm{~F}$ & D & Sí & 3 & 5 & 16 & 134,58 & 0 & 0 & 73 & 270 & 2 \\
\hline 13 & 54 & M & D & Sí & 4 & 7 & 16 & 104,28 & 0 & 0 & 71 & 280 & 2 \\
\hline 14 & 21 & M & D & Sí & 4 & 6 & 18 & 109,37 & 0,8 & 7,5 & 83 & 265 & 2 \\
\hline 15 & 42 & M & I & No & 4 & 5 & 14 & 45,31 & 0,5 & 4,85 & 68 & 264 & 3 \\
\hline 16 & 40 & M & I & No & 5 & 8 & 15 & 114,28 & 0 & 4,6 & 79 & 260 & 2 \\
\hline 17 & 24 & M & D & Sí & 4 & 8 & 17 & 89,52 & 0 & 3,29 & 81 & 258 & 2 \\
\hline 18 & 22 & M & D & Sí & 4 & 10 & 17 & 95,06 & 0 & 0,66 & 107 & 265 & 3 \\
\hline 19 & 49 & M & I & No & 5 & 16 & 16 & 75,63 & 0 & 0 & 67 & 273 & 2 \\
\hline 20 & 54 & $\mathrm{~F}$ & D & Sí & 4 & 3 & 18 & 106,82 & 0 & 4,05 & 61 & 269 & 2 \\
\hline 21 & 34 & $\mathrm{~F}$ & I & No & 4 & 19 & 14 & 100 & 0 & 0,83 & 74 & 263 & 2 \\
\hline 22 & 19 & M & D & Sí & 4 & 7 & 19 & 100 & 0 & 0 & 65 & 265 & 3 \\
\hline 23 & 43 & M & D & Sí & 4 & 6 & 19 & 96,51 & 0 & 0 & 73 & 259 & 2 \\
\hline 24 & 17 & $\mathrm{~F}$ & I & Sí & 4 & 7 & 14 & 101,81 & 0 & 0 & 66 & 268 & 2 \\
\hline
\end{tabular}




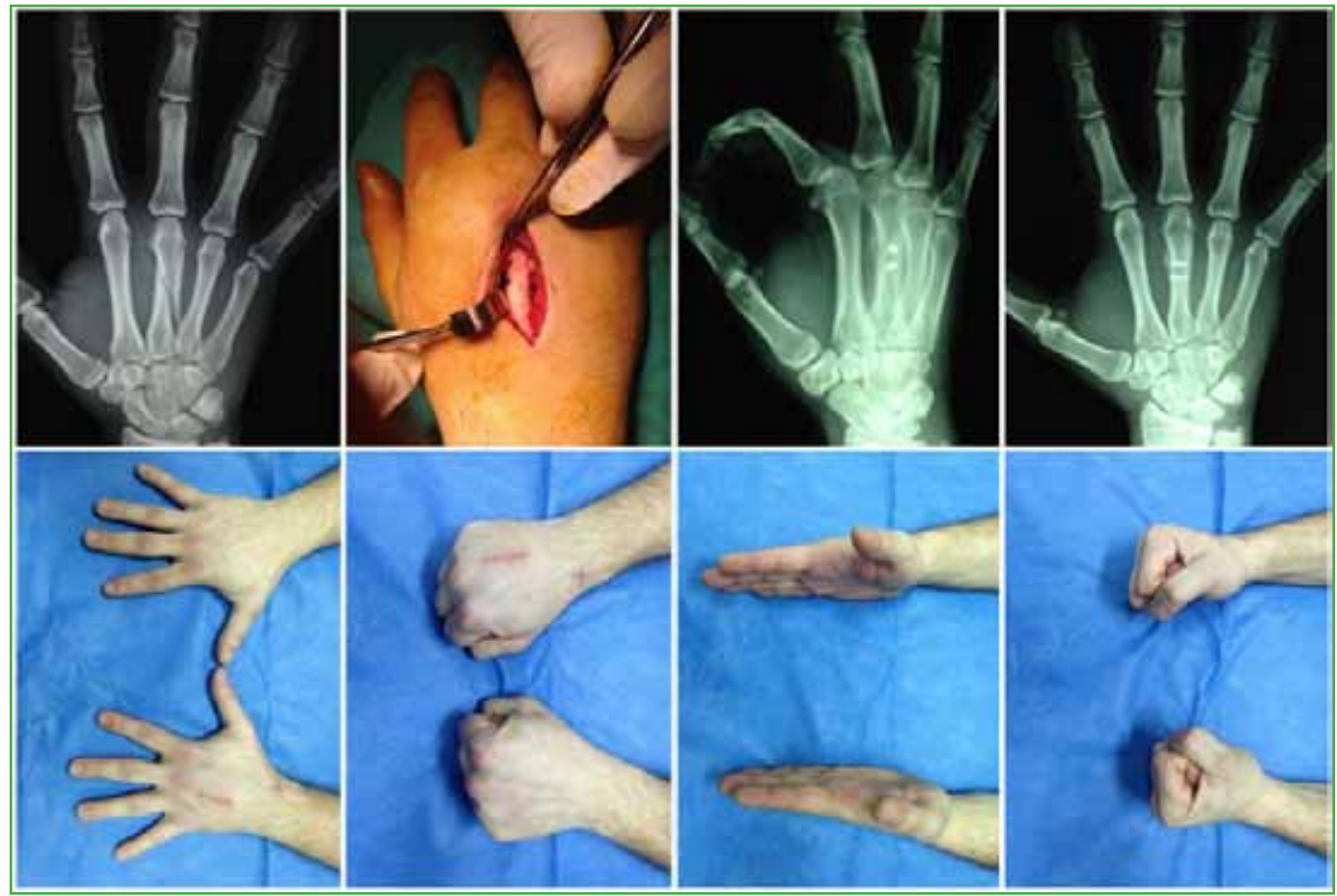

Figura 1. Paciente tratado con tornillos mediante técnica de compresión interfragmentaria. Imágenes preoperatoria, intraoperatoria, posoperatorias y resultado funcional al final del seguimiento.

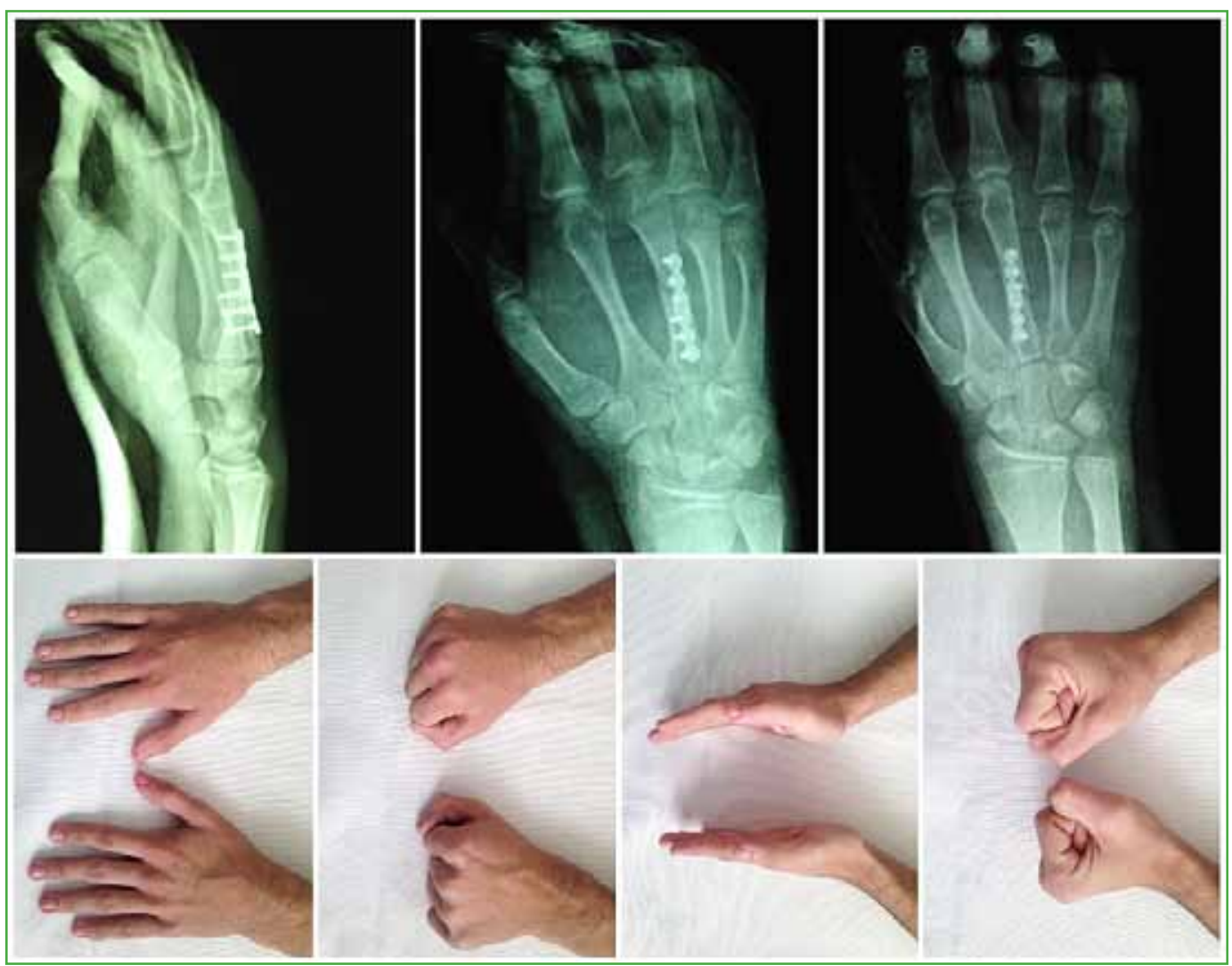

Figura 2. Paciente tratado con placa de osteosíntesis de $2 \mathrm{~mm}$. Imágenes posoperatorias y resultado funcional al final del seguimiento. 
Tabla 2. Serie de pacientes tratados con placas y tornillos

\begin{tabular}{|c|c|c|c|c|c|c|c|c|c|c|c|c|c|}
\hline $\mathbf{N}^{0}$ & Edad & Sexo & $\begin{array}{c}\text { Mano } \\
\text { lesionada }\end{array}$ & $\begin{array}{l}\text { Mano } \\
\text { hábil }\end{array}$ & $\begin{array}{l}\text { Metacar- } \\
\text { piano } \\
\text { afectado }\end{array}$ & $\begin{array}{l}\text { Días } \\
\text { preq }\end{array}$ & $\begin{array}{l}\text { Días de in- } \\
\text { movilización }\end{array}$ & $\begin{array}{c}\% \text { de } \\
\text { fuerza }\end{array}$ & $\begin{array}{c}\text { Dis- } \\
\text { tancia } \\
\text { pulpejo- } \\
\text { palma }\end{array}$ & $\begin{array}{l}\text { Pun- } \\
\text { taje } \\
\text { DASH }\end{array}$ & $\begin{array}{l}\text { Días has- } \\
\text { ta el alta } \\
\text { laboral }\end{array}$ & $\begin{array}{l}\text { Movi- } \\
\text { lidad } \\
\text { activa } \\
\text { total }\end{array}$ & $\begin{array}{l}\text { Complica- } \\
\text { ción }\end{array}$ \\
\hline 1 & 58 & $\mathrm{M}$ & I & No & 5 & 6 & 14 & 89,74 & 2 & 6,8 & 98 & 200 & \\
\hline 2 & 56 & $\mathrm{M}$ & D & Sí & 5 & 8 & 17 & 109,37 & 1 & 7,5 & 89 & 265 & \\
\hline 3 & 49 & $\mathrm{M}$ & D & Sí & 4 & 5 & 16 & 87,5 & 0 & 3,33 & 79 & 266 & \\
\hline 4 & 29 & $\mathrm{M}$ & D & Sí & 5 & 7 & 15 & 88 & 0,5 & 1,72 & 63 & 235 & \\
\hline 5 & 28 & $\mathrm{M}$ & D & Sí & 5 & 9 & 14 & 100 & 0 & 0 & 81 & 271 & \\
\hline 6 & 31 & $\mathrm{M}$ & I & No & 3 & 4 & 15 & 100 & 0 & 0 & 57 & 260 & \\
\hline 7 & 37 & $\mathrm{~F}$ & D & Sí & 4 & 6 & 15 & 100 & 0 & 1,17 & 67 & 258 & \\
\hline 8 & 47 & $\mathrm{~F}$ & I & Sí & 4 & 5 & 19 & 92 & 1 & 2016 & 85 & 241 & Retiro \\
\hline 9 & 32 & $\mathrm{M}$ & D & Sí & 2 & 8 & 16 & 102,56 & 0 & 0 & 71 & 272 & \\
\hline 10 & 42 & $\mathrm{M}$ & I & No & 3 & 7 & 18 & 93,83 & 0 & 0 & 67 & 265 & \\
\hline 11 & 71 & $\mathrm{M}$ & D & Sí & 5 & 11 & 19 & 100 & 2 & 8,31 & & 238 & $\begin{array}{l}\text { Retiro y } \\
\text { tenólisis }\end{array}$ \\
\hline 12 & 35 & $\mathrm{M}$ & I & Sí & 4 & 6 & 14 & 91,57 & 0 & 0 & 73 & 268 & \\
\hline 13 & 43 & $\mathrm{M}$ & D & Sí & 3 & 7 & 16 & 98,79 & 0 & 1,31 & 59 & 259 & \\
\hline 14 & 45 & $\mathrm{M}$ & D & No & 4 & 4 & 17 & 93,9 & 0,5 & 3,36 & 70 & 249 & \\
\hline 15 & 22 & $\mathrm{M}$ & D & Sí & 4 & 10 & 18 & 100 & 0 & 0 & 68 & 266 & \\
\hline 16 & 69 & $\mathrm{~F}$ & D & Sí & 5 & 14 & 15 & 79,24 & 1 & 2,18 & & 237 & \\
\hline 17 & 17 & $\mathrm{M}$ & D & Sí & 2 & 5 & 16 & 98,41 & 0 & 0 & & 273 & \\
\hline
\end{tabular}

$\mathrm{M}=$ masculino, $\mathrm{F}=$ femenino, $\mathrm{I}=$ izquierda, $\mathrm{D}=$ derecha

\section{Seguimiento posoperatorio}

Se indicó una valva palmar antebraquidigital de yeso por un promedio de 16 días (rango 10-19). Luego se retiraron los puntos y se inició el proceso de rehabilitación con terapia ocupacional. Se tomaron radiografías de frente y de perfil antes de la cirugía, en el posoperatorio inmediato y al final del seguimiento. Se consideró que un resultado era satisfactorio si se cumplían los criterios establecidos por Pun y cols. (en metacarpianos, acortamiento $<6 \mathrm{~mm}$ sin rotación ni angulación de la cabeza).

La evaluación subjetiva al final del seguimiento se realizó con el puntaje DASH.

La evaluación objetiva incluyó goniometría, distancia pulpejo-palma y dinamometría. Usando un goniómetro, se registró el rango de movilidad activa total definido como la suma de la flexión activa de las articulaciones metacarpofalángica, interfalángica proximal y distal, menos el déficit de extensión de dichas articulaciones. Se consideró que un resultado era excelente, si era $>150^{\circ}$; bueno, de $125^{\circ}$ a $149^{\circ}$; regular de $90^{\circ}$ a $124^{\circ}$ y malo $<90^{\circ}$.

Se cotejó la distancia pulpejo-palma expresada en centímetros. El resultado se consideró excelente, si la distancia era $\leq 1 \mathrm{~cm}$; bueno, de 1 a $1,5 \mathrm{~cm}$; regular de 1,6 a $3 \mathrm{~cm}$ y malo $>3 \mathrm{~cm}$. 
La evaluación dinamométrica se realizó con dinamómetro hidráulico Baseline®, y se consignó el porcentaje de recuperación de la fuerza respecto del lado contralateral. Se registraron la cantidad de días desde la lesión hasta el reinicio de la actividad laboral habitual y las complicaciones quirúrgicas.

El análisis estadístico se realizó con el programa IBM SPSS Statistics 19.0. Se usó una prueba mediana no paramétrica. Se consideró significativo un valor $\mathrm{p}<0,05$.

\section{RESULTADOS}

El tiempo promedio de seguimiento fue de 24.5 meses (rango 12-43). El grupo 1 (tratado solo con tornillos) tuvo un puntaje DASH posoperatorio promedio de 1,6 (rango $0-4,8$ ), una movilidad activa total promedio de $253,7^{\circ}$ (rango 230-280), una distancia pulpejo-palma promedio de 0,15 cm (rango 0-1) y una recuperación promedio del $97,6 \%$ de la fuerza de puño con respecto a la contralateral. Este grupo de pacientes se reintegró a su actividad laboral habitual en un promedio de 77 días (rango 52-107).

El grupo 2 (tratado con placas y tornillos) tuvo un puntaje DASH promedio de 2,1 (rango 0-8,3), una movilidad activa total promedio de $254,2^{\circ}$ (rango $200-273$ ), una distancia pulpejo-palma de $0,5 \mathrm{~cm}$ (rango $0-2$ ) y dinamometría con recuperación promedio del $96,1 \%$ de la fuerza de puño respecto de la contralateral. Este grupo de pacientes se reintegró a su actividad laboral en un promedio de 68.5 días (rango 57-98).

El análisis estadístico de los resultados no mostró diferencias estadísticamente significativas para: el puntaje DASH $(\mathrm{p}=0,5)$, la movilidad activa total $(\mathrm{p}=0,3)$, la distancia pulpejo-palma $(\mathrm{p}=0,10)$ y dinamometría $(\mathrm{p}=$ $0,38)$.

En cuanto a las complicaciones, no se registraron casos de infección, seudoartrosis, consolidación viciosa, aflojamiento o rotura del implante, lesión vasculonerviosa, ni síndrome doloroso regional complejo. Solo en el grupo 2 (placas y tornillos), hubo dos casos de tenosinovitis extensora relacionada con el implante (posterior al retiro de material de osteosíntesis), sumado, en uno de ellos, a adherencia tendinosa; se procedió a la tenólisis de extensor.

\section{DISCUSIÓN}

Las fracturas diafisarias de los metacarpianos representan una importante proporción de los cuadros traumáticos que se le presentan al cirujano de mano. En este estudio, se comparó, en forma retrospectiva, el resultado funcional después del tratamiento con tornillos interfragmentarios solos o con placas y tornillos.

Sobre la base de estudios biomecánicos previos, los límites tolerables de acortamiento, angulación y rotación de los metacarpianos en las fracturas diafisarias están claramente establecidos. En el contexto de su alta incidencia, un razonamiento válido sería considerar el predominio de la resolución quirúrgica por sobre la incruenta, pero la bibliografía actual se contrapone a esta argumentación, el porcentaje varía entre el 5\% y el 30\% de los casos, con amplia preferencia por las agujas de Kirschner, seguidas, en orden decreciente, de los tornillos solos y excepcionalmente las placas con tornillos. ${ }^{13}$

Ambos sistemas, tornillos solos y placas y tornillos, proveen una reducción anatómica y una fijación estable para comenzar una rehabilitación temprana, en pos de conseguir la restauración completa o próxima al ideal de la mano. ${ }^{1-3,6,8,10,11,13}$

El empleo de tornillos interfragmentarios solos implica la correcta aplicación de la técnica de compresión interfragmentaria. Para su instauración debe cumplirse la premisa de que la longitud del trazo fracturario sea, al menos, dos veces el diámetro del metacarpiano y que el emplazamiento del orificio del tornillo sea perpendicular al trazo. El orificio proximal (canal liso) debe tener el mismo diámetro de rosca del tornillo, ejerciendo un efecto directo sobre la resistencia al arrancamiento (pull-out). El orificio distal clásicamente respeta el diámetro del alma del tornillo, ejerciendo un efecto directo sobre la resistencia a la flexión y la tensión. ${ }^{1,2,6,7,14,15}$ Adams y cols. consideran que la estabilidad axial provista por un solo tornillo interfragmentario es limitada; por ello, la aplicación de dos o más tornillos según la longitud del trazo aporta un incremento de la rigidez del sistema, aunque la fuerza de torsión no sea neutralizada. ${ }^{1}$

Las placas y los tornillos otorgan mayor estabilidad a expensas de un mayor costo biológico producto de la desperiotización. Por el dorso (arbotante de tensión), desde el punto de vista biomecánico, resulta favorable la aplicación de la placa, al resistir la fuerza de flexión fracturaria (arbotante de compresión). Los factores considerados al elegir la placa para la osteosíntesis incluyen el tipo de implante (con bloqueo angular o sin él) y la fijación unicortical o bicortical de los tornillos. ${ }^{1,14,15}$

Prevel y cols., y Cordey y cols. demostraron que las placas bloqueadas en metacarpianos proporcionan más rigidez que las no bloqueadas, ya que son el resultado de la suma de todas las interfaces hueso-tornillo del sistema. 
La falla de las placas no bloqueadas resulta del aflojamiento de tornillos individualmente, mientras que las placas bloqueadas cuando fallan, lo hacen como una unidad. ${ }^{14,15}$

Ochman y cols. estudiaron experimentalmente posibles diferencias de los tornillos unicorticales o bicorticales. En particular, no se registraron diferencias significativas en la rigidez entre los grupos de tornillos unicortical y bicortical con placas sin bloqueo y con bloqueo. ${ }^{16}$

Trevisan y cols. informaron resultados aceptables con una pronta reincorporación laboral al utilizar placas con tornillos, aunque consideraron la frecuente irritación de los tendones extensores debido al abordaje, y también de los flexores relacionado con el exceso de longitud palmar de los tornillos. ${ }^{17}$

Firoozbakhsh y cols. evaluaron las diferencias cuantitativas en la resistencia a la fatiga y la estabilidad frente a cargas cíclicas, en manos cadavéricas donde se reprodujeron fracturas de metacarpianos. Dividieron el tratamiento en placa dorsal y tornillos, dos tornillos dorsales compresivos, bandas de tensión y agujas de Kirschner intramedulares. La placa registró mayor resistencia ante las pruebas de flexión (1,5 veces), torsión (1,6 veces) y carga axial (2,5 veces), respecto a la segunda fijación más fuerte (tornillos dorsales compresivos). ${ }^{18}$

Basar y cols. compararon ambos tratamientos en las fracturas diafisarias de metacarpianos. La placa con tornillos proporcionó una fijación más rígida en comparación con los tornillos solos, permitiendo una mejor fuerza de puño inicial, especialmente beneficiosa en trabajadores manuales, acelerando el reingreso laboral, aunque no se observaron diferencias significativas al final del seguimiento. ${ }^{1}$

En nuestro estudio, consideramos importante mencionar que el abordaje quirúrgico en un plano para intrínsecos y periostio proporciona la ventaja técnica de un cierre cómodo sobre el material de osteosíntesis y su consiguiente menor posibilidad de irritación o adherencia tendinosa. ${ }^{4,6}$ En la comparación de esta serie de pacientes, ambas técnicas resultaron eficaces y reproducibles. Los tornillos interfragmentarios solos representaron un procedimiento menos complejo y de menor exposición; además, no se registraron complicaciones (aunque el tiempo hasta el retorno laboral fue más prolongado). Por su parte, el empleo de placa con tornillos confirió más rigidez al sistema, permitiendo un retorno laboral más precoz, aunque con más complicaciones y costo biológico.

Consideramos que los factores limitantes de este estudio son su diseño retrospectivo, la acotada muestra de los subgrupos, la heterogeneidad del material utilizado y el manejo a cargo de distintos cirujanos. Sin embargo, sus fortalezas son la población con un cuadro homogéneo y el seguimiento mínimo.

\section{CONCLUSIONES}

El tratamiento de los pacientes con fracturas oblicuas o espiroideas largas de metacarpianos, mediante reducción abierta y fijación interna con tornillos solos interfragmentarios o placas y tornillos, logró resultados similares. Se destaca el menor tiempo hasta la reincorporación laboral y la mayor cantidad de complicaciones en el grupo tratado con placas y tornillos.

Conflicto de intereses: Los autores no declaran conflictos de intereses.

\section{BIBLIOGRAFÍA}

1. Adams JE, Miller T, Rizzo M. The biomechanics of fixation techniques for hand fractures. Hand Clin 2013;29:493500. https://doi.org/10.1016/j.hcl.2013.08.004

2. Basar H, Basar B, Bacsi O, Topkar OM, Erol B, Tetik C. Comparison of treatment of oblique and spiral metacarpal and phalangeal fractures with mini plate plus screw or screw only. Arch Orthop Trauma Surg 2015;135(4):499-504. https://doi.org/10.1007/s00402-015-2164-3 
3. Freeland AE, Geissler WB, Weiss AP. Surgical treatment of common displaced and unstable fractures of the hand. Instr Course Lect 2002;51:185-201. PMID: 12064103

4. Freeland AE, Orbay JL. Open reduction and internal fixation of the tubular bones of the hand. En: Strickland JW, Graham T. Master techniques in orthopaedic surgery. The Hand. $2^{\text {nd }}$ ed. Philadelphia: Lippincott Williams \& Wilkins; 2016:3-26.

5. Heim U, Pfeiffer KM. Interfragmental compression with lag screws. En: Heim U, Pfeiffer KM (ed.). Internal fixation of small fractures. Berlin: Springer Verlag; 1988:32-4.

6. Henry MH. Fractures of the proximal phalanx and metacarpals in the hand: preferred methods of stabilization. $J A m$ Acad Orthop Surg 2008;16(10):586-95. https://doi.org/10.5435/00124635-200810000-00004

7. Jeff Justis E. Fracturas luxaciones y lesiones ligamentarias. En: Campbell Cirugía ortopédica, $8^{\mathrm{a}}$ ed. Buenos Aires: Panamericana; 1992:2912-20.

8. Kawamura K, Chung KC. Fixation choices for closed simple unstable oblique phalangeal and metacarpal fractures. Hand Clin 2006;22(3):287-95. https://doi.org/10.1016/j.hcl.2006.02.018

9. Liporace FA, Kinchelow T, Gupta S, Kubiak E, McDonnell M. Minifragment screw fixation of oblique metacarpal fractures: a biomechanical analysis of screw types and techniques. Hand (NY) 2008;3(4):311-5. https://doi.org/10.1007/s11552-008-9108-0

10. Roth JJ, Auerbach DM. Fixation of hand fractures with bicortical screws. J Hand Surg Am 2005;30(1):151-3. https://doi.org/10.1016/j.jhsa.2004.07.016

11. Simonetti L, Boretto J, Galucci G, Sarme A, De Carli P. Fracturas diafisarias de los metacarpianos. Tratamiento con tornillos interfragmentarios. Rev Asoc Arg Ortop Traumatol 2009;74(3):242-8. Disponible en: https://www.aaot.org.ar/revista/2009/n3/Rev_AsocArgentOrtopTraumatol_242Boretto.pdf

12. Stern PJ. Fractures of the metacarpals and phalanges. En: Green DP, Hotchikss RN, Pederson WC, Wolfe SW (eds.). Green's operative hand surgery, $5^{\text {th }}$ ed. Philadelphia: Elsevier Churchill Livingstone; 2005, vol. 1, p. $286-94$.

13. Tang JB, Blazar PE, Giddins G, Lalonde D, Martínez C, Solomons M. Overview of indications, preferred methods and technical tips for hand fractures from around the world. J Hand Surg Eur 2015;40E(1):88-97. https://doi.org/10.1177/1753193414561942

14. Prevel CD, Eppley BL, Jackson JR, Moore K, McCarthy M, Sood R, et al. Mini ad micro plating of phalangeal and metacarpal fractures: a biomechanical study. J Hand Surg Am 1995;20:44-9. https://doi.org/10.1016/s0363-5023(05)80057-7

15. Cordey J, Borgeaud M, Perren SM. Force transfer between the plate and the bone: relative importance of the bending stiffness of the screws friction between plate and bone. Injury 2000;31(3):21-8. https://doi.org/10.1016/s0020-1383(00)80028-5

16. Ochman S, Doht S, Paletta J, Langer M, Raschke M, Meffert R. Comparison between locking and non-locking plates for fixation of metacarpal fractures in an animal model. J Hand Surg Am 2010;35:597-603. https://doi.org/10.1016/j.jhsa.2010.01.002

17. Trevisan C, Morganti A, Casiraghi A, Marinoni E. Low-severity metacarpal and phalangeal fractures treated with miniature plates and screws. Arch Orthop Trauma Surg 2004;124(10):675-80. https://doi.org/10.1007/s00402-004-0745-7

18. Firoozbakhsh K, Moneim M, Howey T, Castaneda E, Pirela-Cruz M. Comparative fatigue strengths and stabilities of metacarpal internal fixation techniques. J Hand Surg Am 1993;18(6):1059-68.

https://doi.org/10.1016/0363-5023(93)90403-P 